\title{
O uso da aspirina para o tratamento da pré-eclâmpsia: uma revisão narrativa
}

\author{
The use of aspirin for the treatment of pre-eclampsia: a narrative review
}

El uso de aspirina para el tratamiento de la preeclampsia: una revisión narrativa

Maria Luiza Andrade Siqueira ${ }^{1 *}$, Stephany Brini de Mendonça ${ }^{1}$, Cecilia Miranda Gonçalves ${ }^{1}$, Mariana Santos Magalhães Cortez ${ }^{1}$, Maria Clara Moreira de Oliveira1, Roberta Cíntia Sousa Coelho' ${ }^{1}$ Milena Melo Gambogi ${ }^{1}$, Michael Zarnowski Passos ${ }^{2}$.

\section{RESUMO}

Objetivo: Esclarecer aspectos da pré-eclâmpsia, suas possíveis complicações e o ef eito da aspirina como forma de tratamento para tal síndrome. Revisão bibliográfica: Obtêm-se o benefício da administração de aspirina em mulheres com fatores de risco, como forma de prevenção da pré-eclâmpsia e da restrição do crescimento fetal, sem potenciais riscos maternos e fetais. A dose de aspirina utilizada para redução da incidência de pré-eclâmpsia varia de 50 a 150mg/dia, a depender do estudo analisado. Entretanto, ao analisar as doses mais recomendadas, podemos inferir que a dosagem mais recomendada e utilizada é a de $100 \mathrm{mg} /$ dia. Considerações finais: A síndrome hipertensiva intercorrente na gestação, conhecida como préeclâmpsia, induz risco de implicações significativas à saúde gestacional e fetal que pode acarretar à morte materna e perinatal ou gerar limitações definitivas na saúde de ambos. Diante disso, obtém-se a importância do estudo de recursos que visam a prevenção, os fatores de risco e as possíveis complicações resultantes de tal condição clínica, como a aspirina.

Palavras-chave: Aspirina, Pré-eclâmpsia, Terapêutica, Farmacologia.

\section{ABSTRACT}

Objective: To clarify aspects of pre-eclampsia, it's possible complications and the effect of aspirin as a form of treatment for this syndrome. Bibliographic review: The benefit of aspirin administration in women with risk factors is obtained, as a way to prevent pre-eclampsia and fetal growth restriction, without potential maternal and fetal risks. The dose of aspirin used to reduce the incidence of pre-eclampsia ranges from 50 to $150 \mathrm{mg} /$ day, depending on the study analyzed. However, when analyzing the most recommended doses, we can infer that the most recommended and used dose is $100 \mathrm{mg} /$ day. Final considerations: The intercurrent hypertensive syndrome in pregnancy, known as pre-eclampsia, induces a risk of significant implications for gestational and fetal health, which can lead to maternal and perinatal death or generate definite limitations on the health of both. Therefore, it is important to study resources aimed at prevention, risk factors and possible complications resulting from such a clinical condition, such as aspirin.

Key words: Aspirin-like agents, Pre-eclampsia, Therapeutics, Pharmacology.

\section{RESUMEN}

Objetivo: Aclarar aspectos de la preeclampsia, sus posibles complicaciones y el efecto de la aspirina como forma de tratamiento de este síndrome. Revisión bibliográfica: Se obtiene el beneficio de la administración de aspirina en mujeres con factores de riesgo, como forma de prevenir la preeclampsia y la restricción del

\footnotetext{
${ }^{1}$ Faculdade de Minas - Belo Horizonte (FAMINAS-BH), Belo Horizonte - MG.

* E-mail: marialuizaandradeif@gmail.com

${ }^{2}$ Hospital das Clínicas (HC-UFMG), Belo Horizonte - MG.
}

SUBMETIDO EM: 9/2021 
crecimiento fetal, sin potenciales riesgos maternos y fetales. La dosis de aspirina utilizada para reducir la incidencia de preeclampsia varía de 50 a $150 \mathrm{mg} / \mathrm{d}$ áa, según el estudio analizado. Sin embargo, al analizar las dosis más recomendadas, podemos deducir que la dosis más recomendada y utilizada es de $100 \mathrm{mg} / \mathrm{d} i ́ a$. Consideraciones finales: El síndrome hipertensivo intercurrente en el embarazo, conocido como preeclampsia, induce el riesgo de implicaciones importantes para la salud gestacional y fetal que puede provocar la muerte materna y perinatal o generar restricciones finales en la salud de ambos. Porlo tanto, se entiende la importancia de los estudios de recursos destinados a la prevención, los factores de riesgo y las posibles complicaciones derivadas de esta condición clínica, como la aspirina.

Palabras clave: Aspirina, Preeclampsia, Terapéutica, Farmacología.

\section{INTRODUÇÃO}

A pré-eclâmpsia é caracterizada como uma síndrome na qual há proteinúria associada a valores de pressão sistólica maiores ou iguais a $140 \mathrm{mmHg}$ e/ou os valores de pressão diastólica iguais ou superiores a $90 \mathrm{mmHg}$, após 20 semanas de gestação em mulheres previamente normotensas, em duas medições seguidas. A síndrome pode cursar com disfunção hepática, neurológica e renal, sendo uma das principais causas de mortalidade e morbidade materna e fetal (GAN J, et al., 2016; MURTONIEMI K, et al., 2018; ROBERGE S, et al., 2018a).

A etiologia da pré-eclâmpsia ainda é desconhecida, mas acredita-se que a placentação defeituosa faça parte da fisiopatologia, estando associada à gravidade e início precoce (MURTONIEMI K, et al., 2018). Desse modo, há evidências de que há diminuição da perfusão uteroplacentária, advinda de disfunção endotelial, agregação plaquetária e ativação da cascata de coagulação. Com isso, pode ocorrer hipóxia e isquemia placentária, que levam a complicações graves para a mãe e para o feto, o qual pode nascer prematuro e com baixo peso (GAN J, et al., 2016; ROLNIK DL, et al., 2017).

Alguns fatores de risco para o desenvolvimento de pré-eclâmpsia são primiparidade, gravidez préeclâmptica anterior, doença renal crônica, gravidez múltipla, diabetes mellitus, lúpus eritematoso sistêmico, idade materna maior que 40 anos, entre outros conforme o American College of Obstetricians and Gynecologists e o National Institute for Health and Clinical Excellence, do Reino Unido (MONE F, et al., 2016).

Diversos estudos têm avaliado sobre o benefício da administração de aspirina em mulheres com fatores de risco, como forma de prevenção da pré-eclâmpsia e da restrição do crescimento fetal, sem potenciais ef eitos adversos. A recomendação, conforme os recentes estudos, é que o uso da medicação deve ocorrer em baixas dosagens, sendo iniciado antes de 16 semanas gestacionais (STANESCU AD, 2018).

A aspirina, ou ácido acetilsalicílico, atua por meio da inibição da cicloxigenase, impedindo, assim, a formação de prostaglandinas e tromboxano, ou seja, possui propriedades anti-inflamatórias e de inibição da agregação plaquetária. Além disso, sua atuação no tromboxano inibe a vasoconstrição plaquetária, sendo benéfica em sua atuação na etiologia já descrita da pré-eclâmpsia (HAMULYÁK EN, et al., 2020; GAN J, et al., 2016).

Outrossim, o fator de crescimento placentário (PIGF, do inglês Placental Growth Factor), que é membro da família dos fatores de crescimento vascular e é secretado majoritariamente por trofoblastos durante 0 período gestacional, tem seu papel na gestação cada vez mais estudado. Isso porque pode estar envolvido com o crescimento e diferenciação da placenta, além de estar relacionado com um fator pró-angiogênico, tirosina quinase-1 tipo Feline McDonough Sarcoma ( $\mathrm{fms}$ ) solúvel, fator de crescimento do endotélio vascular e sua contraparte antiangiogênica, os quais fazem parte de um sistema responsável por manter a estrutura e função endotelial normal (MURTONIEMI K, et al., 2018; O'GORMAN N, et al., 2016).

Nesse sentido, os níveis séricos de PIGF aumentam durante a gestação, com pico em 30 semanas, aproximadamente. Entretanto, em mulheres com pré-eclâmpsia, os níveis desse fator diminuem em todo o período gestacional, levando à inadequada angiogênese placentária. $O$ uso da aspirina em baixas dosagens 
e sua ação no PIGF têm sido estudados por Panagodage S, et al. (2016), em um estudo in vitro, no qual é demonstrado que o Ácido Acetilsalicílico (AAS) pode promover a modulação da produção de citocinas, o que atenua a apoptose eleva a um crescimento adequado de trofoblastose, em consonância, melhora sua função, levando a uma maior secreção do PIGF. Desse modo, há maior regulação dos processos supracitados (MURTONIEMI K, et al.,2018; PANAGODAGE S, et al., 2016).

Dessa forma, o objetivo do artigo foi realizar uma revisão bibliográfica acerca do uso da aspirina no tratamento da pré-eclâmpsia, descrevendo as alterações fisiológicas e biomarcadores presentes no processo, a dose, posologia e duração do tratamento com o respectivo fármaco, além de outros fatores que podem interferir no tratamento.

\section{REVISÃO BIBLIOGRÁFICA}

\section{Alterações fisiológicas da gravidez e biomarcadores}

O corpo da mulher passa por diversas alterações durante a gravidez para comportar o feto durante os 9 meses seguintes e para suprir sua própria demanda de energia. Porém, as mudanças fisiológicas que ocorrem na gestação também podem ser responsáveis por alterações farmacocinéticas de medicações, como a aspirina, que após passar por inúmeras pesquisas, em certas doses seria capaz de modular a produção de citocinas e aumentar a secreção de trofoblastos, protegendo contra a vasoconstrição placentária, a coagulação sanguínea e a malformação da artéria espiralada. Por conseguinte, a medicação tem sido recomendada para a prevenção da pré-eclâmpsia em mulheres grávidas de alto risco (SHANMUGALINGAM R, et al., 2019; PANAGODAGE S, et al., 2016; GAN J, et al., 2016). Além de reduzir as possibilidades da préeclâmpsia, a aspirina apresenta outros benefícios, como a redução do crescimento intrauterino restrito e nascimentos pré-termos (GAN J, et al., 2016).

De acordo com Murtoniemi K, et al. (2018), foram realizados estudos que demonstraram que, durante o período da gravidez, a concentração sanguínea do fator de crescimento placentário é reduzida desde o começo da gestação em mulheres que posteriormente desenvolverão a pré-eclâmpsia. Em gestações normais, os níveis séricos do PIGF se encontram aumentados na circulação materna no primeiro e segundo trimestre da gestação, apresentando um valor mais alto no decorrer das 30 semanas e diminuindo em seguida.

A função do PIGF é desconhecida, porém ele faz parte dos fatores de crescimento vascular e é secretado durante a gestação pelos trofoblastos. Em conjunto com outro fator pro-angiogênico, o fator de crescimento do endotélio vascular, sua contraparte antiangiogênica e a tirosina quinase-1 tipo fms solúvel (sFlt-1)- que possui propriedades antiangiogênicas-estariam conectados a um sistema complexo que conserva a estrutura e a função endotelial normal. Dessa forma, o PIGF funciona como uma espécie de biomarcador eficaz para diagnosticar precocemente esta condição patológica, sobretudo quando associado com outros biomarcadores e medições biofísicas (MURTONIEM K, et al., 2018).

Até o momento, não se sabe a causa da pré-eclâmpsia, porém existem grandes indícios que a placentação prejudicada também esteja ligada a fisiopatologia, em conjunto com a antecipação e o estado crítico da doença (MURTONIEMI K, et al., 2018). Portanto, fisiologicamente explicando a sub perfusão uteroplacentária da pré-eclâmpsia, pressupõe-se que o fluxo sanguíneo da placenta seja reduzido devido à ativação da cascata de coagulação, além da agregação placentária e da disfunção endotelial, que tem correlação com a mediação através de prostaciclina e tromboxano A2 (GAN J, et al., 2016).

\section{Uso da aspirina $\mathrm{x}$ heparina}

Nos estudos utilizados para a síntese desse artigo pouco foi abordado sobre a interferência da heparina na pré-eclâmpsia e na redução de perda do feto, embora ela seja utilizada em gestantes com síndrome do anticorpo antifosfolipídeo, em associação a aspirina. Tal síndrome configura-se como risco à mãe e ao feto, pois o sistema imunológico age de modo a atacar células sanguíneas, causando coágulos p or todo o sistema circulatório, inclusive em órgãos (HAMULYÁK EN, et al., 2020). 
Artigos revisionais de ensaios randomizados e clusters mostram a eficácia da heparina e da aspirina em conjunta ação durante o período gestacional de mulheres que têm a síndro me do anticorpo antifosfolipídeo, aumentando o número de nascidos vivos. Em contrapartida, as evidências do uso da aspirina em associação com a heparina referente aos estudos utilizados foram escassas, justificadas por baixa disponibilidade de gestantes que se submetem a fazer uso de ambas as medicações em pesquisas, além de um certo preconceito à terapêutica. Portanto, é inconclusiva a ação benéfica da heparina com aspirina em situações de pré-eclâmpsia (HAMULYÁK EN, et al., 2020; LECARPENTIER E, et al., 2018).

\section{Tratamento profilático da Pré-eclâmpsia com aspirina: dose, posologia e duração}

Nos artigos utilizados houve uma variação no que tange à dose de aspirina eficaz para prevenção da préeclâmpsia: seis deles fazem referência à dose igual ou superior a $100 \mathrm{mg} /$ dia ou sugerem intervalos como 50 a $100 \mathrm{mg} / \mathrm{dia}, 75$ a $150 \mathrm{mg} / \mathrm{dia}$ e 80 a $150 \mathrm{mg} / \mathrm{dia}$ (MURTONIEMI K, et al., 2018; ROBERGE S, et al., 2018a; ROBERGE S, et al., 2018b; RONILK DL, et al., 2017; SHANMUGALINGAM R, et al., 2019; STANESCU AD, et al., 2018).

Um artigo refere dose de $75 \mathrm{mg} /$ dia e outro cita um intervalo de 60 a $80 \mathrm{mg} / \mathrm{dia}$ de acordo com algumas associações profissionais, enquanto um dos artigos não faz menção a uma dose determinada (GAN J, et al., 2016; RONILK DL, et al., 2017; O'GORMAN N, et al., 2016).

A dose de $75 \mathrm{mg}$ demonstrou eficácia, porém, o ef eito profilático da pré-eclâmpsia com aspirina é dosedependente (GAN J, et al., 2016, RONILK DL, et al., 2017). Alguns pacientes podem apresentar níveis muito baixos dos metabólitos da aspirina, demonstrando que a dose está ineficaz ou que a paciente não está aderindo adequadamente ao tratamento (NAVARATNAM K, et al., 2019). Dessa forma, deve-se optar por uma dose maior, porém segura, como $100 \mathrm{mg}$, a qual é a mais utilizada, pertencendo aos intervalos dispostos na tabela, sendo que essa dose pode chegar até $150 \mathrm{mg}$ (ROBERGE S, et al., 2018a, SHANMUGALINGAM R, et al., 2019; RONILK DL, et al., 2017; STANESCU AD, et al., 2018).

Outrossim, a gravidez promove alterações fisiológicas, como o aumento da taxa de depuração: quando mulheres grávidas em uso de $150 \mathrm{mg}$ de aspirina foram comparadas com mulheres adultas, não grávidas, em uso de $100 \mathrm{mg}$ de aspirina, observou-se que a quantidade de metabólitos do respectivo fármaco nas mulheres grávidas foi praticamente igual àquela encontrada no outro grupo de mulheres, fato este que corrobora para a necessidade do ajuste da dose de aspirina usada durante a gravidez para obtenção dos ef eitos desejáveis e precaução dos eventos adversos (SHANMUGALINGAM R, et al., 2019).

Em relação à posologia, foi demonstrado que o melhor horário para consumo da aspirina a fim de aumentar a sua eficácia e obtenção do ef eito profilático da pré-eclâmpsia, assim como parto prematuro e crescimento fetal restrito foi à noite, logo antes de dormir (STANESCU AD, et al., 2018).

O início do tratamento profilático com aspirina também é um fatorimportante a ser considerado: os estudos demonstram que a aspirina deve ser iniciada antes ou em até 16 semanas de gestação, sendo que em alguns casos, o intervalo para início foi entre 11 e 14 semanas (MURTONIEMI K, et al., 2018; RONILK DL, et al., 2017, ROBERGE S, et al., 2018a; ROBERGE S, et al., 2018b; O'GORMAN N, et al., 2016; STANESCU AD, et al., 2018).

A idade gestacional é importante, visto que o consumo de aspirina após a 16 semana de gestação não demonstrou um efeito significativo na redução da incidência da pré-eclâmpsia devido ao fato do desenvolvimento da placenta já estar concluído por volta da $18^{\circ}$ semana, tornando assim o mecanismo de ação da aspirina basicamente isento de benefícios adicionais (O'GORMAN N, et al., 2016; ROBERGE S, et al., 2018b). Em contrapartida, uma metanálise realizada com 17 estudos clínicos randomizados demonstrou redução significativa da incidência de pré-eclâmpsia mesmo nas mulheres nulíparas que iniciaram 0 tratamento profilático com aspirina após a 16 semana de gestação (ANDRIKOPOULOU M, et al., 2018).

Já no que se refere à interrupção do consumo da aspirina, este deve ser feito até a $37^{a}$ semana de gestação, pois o uso deste medicamento após essa idade gestacional poderá promover alterações na coagulabilidade do feto, podendo colocar sua vida em risco (STANESCU AD, et al., 2018). 


\section{Grupos que se beneficiariam do uso profilático da aspirina}

Alguns estudos demonstram que a hipertensão crônica com a pré eclâmpsia tem sido considerada como fator de risco para Descolamento Prematuro de Placenta (DPP) e que a placentação prejudicada é um dos fatores fisiopatológicos com grandes graus de evidências no desenvolvimento da pré-eclâmpsia, sendo que fora demonstrado um benefício do uso da aspirina neste grupo específico. Ademais, segundo o American College of Obstetric é recomendado o uso da aspirina em mulheres com histórico de pré-eclâmpsia recorrente ou grave e para profilaxia em gestação gemelar. Dessa forma, os estudos mais recentes e relevantes encontrados abordam esses dois grupos que se beneficiariam do uso profilático da aspirina: gestantes com risco de descolamento prematuro de placenta e em gestação gemelar com níveis aumentados de hCG. (MURTONIEMI K, et al., 2018; EUSER AG, et al., 2016).

Ao relacionar o desenvolvimento de pré-eclâmpsia com o DPP, a Febrasgo coloca a pré-eclâmpsia como fatores de risco maternos em gestações anteriores para DPP, na qual pesquisas anteriores pontuam que tal risco pode ser aumentado de 2.8 para 7.7 vezes. Tendo em vista esse fato, pesquisadores questionaram sobre a possibilidade da profilaxia por aspirina da pré-eclâmpsia ter efeito significativo em casos de descolamento prematuro de placenta. Alguns estudos anteriores relatam que o uso da aspirina como prevenção na pré-eclâmpsia não possuía efeitos importantes na DPP, além de apontarem para ef eitos negativos dessa prática (HADDAD B, et al., 2016; MURTONIEMI K, et al., 2018).

Um ensaio clínico randomizado demonstrou que, como uso da aspirina na dose de $60 \mathrm{mg}$ por dia no intuito da prevenção de pré-eclâmpsia, tem-se um ef eito antiplaquetário associado a um aumento nos números de descolamento de placenta. Porém, algumas metanálises e ensaios clínicos randomizados mais recentes estudaram a relação Idade Gestacional (IG) x dose medicamentosa, nos quais constataram que a dose de $100 \mathrm{mg}$ pordia, com início terapêutico anterior a 16 semanas de gestação, resultou em números consideráveis de mudanças no risco de descolamento de placenta e hemorragia no anteparto, sendo que os limites de eficácia se baseiam nesses critérios de IG e dose da aspirina (ROBERGE S, et al., 2018b)

Dessa forma, como resultado deste estudo, observou-se que uma dose de aspirina menor que $100 \mathrm{mg}$ por dia não influencia no risco de descolamento prematuro da placenta ou hemorragia anteparto, independentemente da idade gestacional no início da terapia. Não obstante, se administrado $100 \mathrm{mg}$ por dia, pode ocorrer um benefício de redução no risco de descolamento prematuro da placenta ou hemorragia anteparto se comparado às mulheres que iniciaram o tratamento com 16 semanas de gestação e aquelas que iniciaram acima de 16 semanas (ROBERGE S, et al., 2018b)

Somando-se a isso, outra relação bem esclarecida em estudos é a associação entre os níveis elevados de Gonadotrofina coriônica humana ( $\mathrm{hCG}$ ) e o desenvolvimento de pré-eclâmpsia. Isso pode se justificar pelo fato de que a elevação da forma clivada de hCG se deve ao aumento da ativação leucocitária nas gestantes com pré-eclâmpsia. Ademais, esse risco está relacionado a mulheres com gestações múltiplas. Assim, foi feita uma pesquisa com 688 mulheres com gestações múltiplas: em $47 \%$ das mulheres era aplicado a aspirina e em $53 \%$ um placebo em uma média gestacional de 21,7 semanas. Não houve diferença estatística relevante entre aspectos como idade, raça e paridade. A incidência geral de pré-eclâmpsia em mulheres foi de $11 \%$ em gestação gemelar e $6 \%$ em gestação gemelar com tratamento profilático com a aspirina. Já a incidência de pré-eclâmpsia em pacientes com alto índice de hCG foi de 15\% e associado ao tratamento profilático foi de 6\% (EUSER AG, et al., 2016).

Desta forma, identificar grupos populacionais que se beneficiariam do uso da aspirina como profilaxia na pré-eclâmpsia e suas doses recomendadas é válido para proporcionar à área médica uma melhor condução clínica. Assim, a conclusão retirada das pesquisas expostas estão relacionadas a dose e população alvo: valores inferiores a 100mg por dia não produzem impacto significativo na redução da pré-eclâmpsia e, quando iniciado antes de 16 semanas de gestação, podem aumentar o risco de DPP e a aspirina proporcionou uma redução na incidência de pré-eclâmpsia em mulheres com gestações gêmeas, sendo que quando relacionado com níveis elevados de hCG essa prevenção se mostrou ainda mais acentuada (ROBERGE S, et al., 2018b; EUSER AG, et al., 2016). 


\section{CONSIDERAÇÕES FINAIS}

Estudos evidenciam a aspirina como uma medicação que com a posologia correta auxilia pré-eclâmpsia, atuando contra a vasoconstrição placentária, a coagulação sanguínea e má formação da artéria espiralada. Infere-se a importância da abordagem do tema devido ao alto número de gestantes acometidas pela síndrome hipertensiva intercorrente na gestação e suas consequências fatais que poderiam ser evitadas pelo uso profilático com o AAS, quando na posologia adequada, condições de risco da gestante pré-determinadas e idade gestacional correta.

\section{REFERÊNCIAS}

1. ANDRIKOPOULOU $\mathrm{M}$, et al. Low-dose aspirin is associated with reduced spontaneous preterm birth in nulliparous women. Am J Obstet Gynecol, 2018; 219 (4): 399.e1-399.e6.

2. EUSER AG, et al. Low-dose aspirin for pre-eclampsia prevention in twins with elevated humanchorionic gonadotropin. Journal of Perinatology, 2016;36 (8): 601-605.

3. GAN J, et al. Preventing preeclampsia and its fetal complications with low-dose aspirin in East Asians and non-East Asians: a systematic review and meta-analysis. Hypertension In Pregnancy, 2016;35(3):426-435.

4. HADDAD B, et al. Enoxaparin and Aspirin Compared with Aspirin Alone to Prevent Placenta-Mediated Pregnancy Complications: A Randomized Controlled Trial. Obstetrics \& Gynecology, 2016;128:1053-63.

5. HUMULYÁK EN, et al. Aspirin or heparin or both for improving pregnancy outcomes in women with persistent antiphospholipid antibodies and recurrent pregnancy loss. The Cochrane database of systematic reviews, 2020;5(5).

6. LECARPENTIER E, et al. Angiogenic Factor Profiles in Pregnant Women with a History of Early-Onset Severe Preeclampsia Receiving Low-Molecular-Weight Heparin Prophylaxis. Obstetrics \& Gynecology, 2018;131 (1): 63-69.

7. MURTONIEMI K, et al. The effect of low-dose aspirin on serum placental growth factor levels in a high-risk PREDO cohort. Pregnancy Hypertension, 2018; 13: 51-57.

8. NAVARATNAM K, et al. Evaluation of agreement of placental growth factor (PIGF) tests and the soluble FMS-like tyrosine kinase 1 (sFlt-1)/PIGF ratio, comparison of predictive accuracy for pre-eclampsia, and relation to uterine artery Doppler and response to aspirin. J Matern Fetal Neonatal Med, 2019; 32 (2): 179-187.

9. O'GORMAN N, et al. Study protocol for the randomised controlled trial: combined multimarker screening and randomised patient treatment with ASpirin for evidence-based PREclampsia prevention (ASPRE). BMJ Open, 2016; 6: 011-801.

10. PANAGODAGE S, et al. Low-Dose Acetylsalicylic Acid Treatment Modulates the Production of Cytokines and Improves Trophoblast Function in an in Vitro Model of Early-Onset Preeclampsia. The American Journal of Pathology, 2016;186(12):3217-3224.

11. ROBERGE S, et al. Aspirin for the prevention of preterm and term preeclampsia: systematic review and metaanalysis. Am J Obstet Gynecol, 2018a;218 (3): 287

12. ROBERGE S, et al. Meta-analysis on the effect of aspirin use for prevention of preeclampsia on placental abruption and antepartum hemorrhage. American Journal Of Obstetrics And Gynecology, 2018b;218 (5): $483-489$.

13. ROLNIK D, et al. Aspirin versus Placebo in Pregnancies at High Risk for Preterm Preeclampsia. New England Joumal of Medicine, 2017;377 (7): 613-622.

14. SHANMUGALINGAM R, et al. A pharmacokinetic assessment of optimal dosing, preparation, and chronotherapy of aspirin in pregnancy. Am J Obstet Gynecol, 2019; 221(3):255.e1-255.e9.

15. STANESCU AD, et al. Low dose aspirin for preventing fetal growth restriction: a randomised trial. Journal Of Perinatal Medicine, 2018;46 (7): 776-779. 